\title{
Ground States to the Generalized Nonlinear Schrödinger Equations with Bernstein Symbols
}

\author{
Jinmyoung Seok ${ }^{1, *}$ and Younghun Hong ${ }^{2}$ \\ ${ }^{1}$ Department of Mathematics, Kyonggi Univeristy, Suwon 16227, Korea \\ 2 Department of Mathematics, Chung-Ang University, Seoul 06974, Korea
}

Received 18 August 2020; Accepted (in revised version) 27 September 2020

Dedicated to Prof. Paul H. Rabinowitz with admiration on the occasion of his 80th birthday

\begin{abstract}
This paper concerns with existence and qualitative properties of ground states to generalized nonlinear Schrödinger equations (gNLS) with abstract symbols. Under some structural assumptions on the symbol, we prove a ground state exists and it satisfies several fundamental properties that the ground state to the standard NLS enjoys. Furthermore, by imposing additional assumptions, we construct, in small mass case, a nontrivial radially symmetric solution to gNLS with $H^{1}$-subcritical nonlinearity, even if the natural energy space does not control the $H^{1}$-subcritical nonlinearity.
\end{abstract}

Key Words: Generalized NLS, solitary waves, variational methods, Bernstein symbols.

AMS Subject Classifications: 35J35, 35J61

\section{Introduction}

We consider the generalized nonlinear Schrödinger equation (NLS for abbreviation) of the form

$$
i \partial_{t} \psi=P(-\Delta) \psi-|\psi|^{p-1} \psi,
$$

where $\psi=\psi(t, x): \mathbb{R} \times \mathbb{R}^{d} \rightarrow \mathbb{C}$ with $d \geq 2$ and $P(-\Delta)$ is defined as a Fourier multiplier of symbol $P=P(\lambda):[0, \infty) \rightarrow \mathbb{R}$. The nonlinear Schrödinger equation is a universal equation describing dynamics of waves in various physical contexts. When $P(\lambda)=\lambda$, the equation is simply the standard NLS, and it appears in nonlinear optics or as a meanfield approximation to the many-body bosonic system The case $P=\lambda^{s / 2}, 0<s<2$ arises in the study of fractional Schrödinger equations [15]. When relativistic effects are taken in account, the symbol $P=\sqrt{\lambda+m^{2}}-m$, or more generally $P=\left(\lambda+m^{2}\right)^{s / 2}-m^{s}$ with $0<s<2$, is chosen [18].

*Corresponding author. Email addresses: jmseok@kgu.ac.kr (J. Seok), yhhong@cau.ac.kr (Y. Hong) 
In this article, we are concerned with the ground state solution to the generalized NLS (1.1). By a ground state, we mean a standing wave solution of the form

$$
\psi(t, x)=e^{i \mu t} u(x), \quad \mu>0,
$$

which minimizes the value of the action integral. A rigorous definition of a ground state shall be given in Section 4 . As for the standard NLS, i.e., the case $P(\lambda)=\lambda$, inserting the standing wave ansatz (1.2) into (1.1), we get the standard stationary NLS

$$
-\Delta u+\mu u=|u|^{p-1} u \text {. }
$$

In this case, the theory of ground states has been almost completed during several decades. A criteria for their existence and nonexistence, depending on the range of $\mu$ and $p$, is established in $[1,22,23]$. Qualitative properties of ground states, such as positiveness, radial symmetry, monotonicity, and uniqueness have been proved in $[4,13,14]$.

With a general symbol $P$, the stationary generalized NLS is given by

$$
P(-\Delta) u+\mu u=|u|^{p-1} u \text { in } \mathbb{R}^{d} .
$$

For some special choices of $P$, such as $\lambda^{s / 2}$ and $\sqrt{\lambda+m^{2}}-m$, a great deal of intensive works on ground states to (1.4) have been carried out. An important remark is that ground states to (1.4) with aforementioned symbols share common qualitative properties such as sign-definiteness, radial symmetry, monotone decreasing property and uniqueness $[3,5,6,9-11,16,20]$.

In this paper, we are interested in finding general conditions on the symbol $P$ which allow ground states to the generalized NLS (1.4) to have the same kinds of qualitative properties. We propose the following structural assumptions for the symbol $P$ :

(H1) $P:[0, \infty) \rightarrow[0, \infty)$ is continuous on $[0, \infty)$ and smooth on $(0, \infty)$;

(H2) $P$ is a Bernstein function, i.e., $P^{\prime}$ is totally monotone (see Section 2 for definition);

(H3) there exists $s \in(0,2]$ such that $P(\lambda) \gtrsim \lambda^{\frac{s}{2}}$ for all large $\lambda$.

Important examples of differential operators satisfying the assumptions (H1)-(H3) include the factional Laplacians $P(\lambda)=\lambda^{s / 2}$ with $0<s<2$ and the generalized pseudorelativistic operators $P=\left(\lambda+m^{2}\right)^{s / 2}-m^{s}$ with $0<s<2$. Some algebraic functions $\frac{\lambda}{(\lambda+1)^{s}}, 0<s<1$ or $\frac{\lambda^{\beta}-1}{\lambda^{\alpha}-1}-1,0<\alpha<\beta<1$ are also included. For more examples satisfying $(\mathrm{H} 1)-(\mathrm{H} 3)$, we refer to the comprehensive book [21].

Our first theorem states that by assuming (H1)-(H3), one can construct a ground state to (1.4) that fulfills desired qualitative properties.

Theorem 1.1 (Existence of a ground state). Suppose (H1)-(H3). Let $p \in(1,(d+s) /(d-s))$ be given. Then for any $\mu>0$, the generalized NLS

$$
P(-\Delta) u+\mu u=|u|^{p-1} u \text { in } \mathbb{R}^{d}
$$

possesses a ground state $u \in H_{P+\mu}$ which is positive, radially symmetric and monotone decreasing in the radial direction. 
The sign-definiteness of a ground state is naturally expected for the original NLS as a result of Hopf maximum principle enjoyed by second order elliptic PDEs. Here, we show that the minimizing property of a ground state and the strict positiveness of the fundamental solution of the operator $P(-\Delta)+\mu$ gives the same consequence.

Theorem 1.2 (Sign-definiteness of a ground state). Suppose $(\mathrm{H} 1)-(\mathrm{H} 3)$. Let $p \in(1,(d+$ $s) /(d-s))$ be given and $u \in H_{P_{u}}$ be a ground state to (1.4). Then $u_{0}$ is either positive everywhere or negative everywhere.

The radial symmetry of a ground state is usually shown by the moving plain method [13] for standard NLS or by strict Riesz rearrangement inequality [17] for fractional NLS and pseudo-relativistic NLS. These methods do not seem to work without the explicit form of $P$. As for uniqueness, a standard argument requires spectral information for the linearized operator at the ground state (see [4]). In [16], Lenzmann showed by a perturbative method that a (radial) ground state to pseudo-relativistic nonlinear Hartree equations (NLH for abbreviation) is unique for the small mass case. In [8], the authors developed, in a similar point of view, some perturbative arguments that show the uniqueness, up to a translation, of a (possibly non-radial) ground state to higher-order NLH. In the same spirit, we are able to show the symmetry and uniqueness of a ground state under more restrictive assumptions on $P$ and $p$ when $\mu$ is sufficiently small. More precisely, we require that

$$
P(0)=0, \quad P^{\prime}(0)=1 \quad \text { and } \quad P^{\prime \prime}(0) \text { exists. }
$$

We note that the choices of $P(0)=0$ and $P^{\prime}(0)=1$ in (H4) are just for numerical simplicity. Indeed, the assumption (H4) can be relaxed to

$$
P(0)>-\mu, \quad P^{\prime}(0)>0 \text { and } \quad P^{\prime \prime}(0) \text { exist }
$$

by a simple reformulation of Eq. (1.4).

Theorem 1.3 (Uniqueness of a ground state). Suppose (H1)-(H4). Let $p \in(1, d /(d-s))$ be given and $u \in H_{P_{\mu}}$ be a positive ground state to (1.4). Then there exists $\mu_{0}>0$ such that if $\mu \in\left(0, \mu_{0}\right)$ then $u$ is unique up to a translation.

The uniqueness result asserted in Theorem 1.3 is perturbative in nature. Indeed, the smallness of the mass $\mu$ is transferred to the smallness of $\epsilon>0$ in the equation

$$
\frac{1}{\epsilon} P(-\epsilon \Delta) v+v=|v|^{p-1} v
$$

by the scaling $u(x)=\epsilon^{\frac{1}{p-1}} v(\epsilon x)$. This shows Theorem 1.3 crucially depends on the existence of $P^{\prime}(0)$ in $(\mathrm{H} 4)$. Actually, the convergence of the formal limit

$$
\frac{1}{\epsilon} P(-\epsilon \Delta) \rightarrow \Delta \quad \text { as } \epsilon \rightarrow 0
$$


plays an indispensable role in the proof.

It is worth to mention that when $P(\lambda)=\sqrt{\lambda+m^{2}}-m$, the formal limit process (1.6) is called the non-relativistic limit. In [6], the authors are able to construct a nontrivial radial solution to pseudo-relativistic NLS (the case that $P(\lambda)=\sqrt{\lambda+m^{2}}-m$ ) with every $H^{1}$ subcritical range of $p$ for sufficiently small $\epsilon>0$ by exploiting the convergence of nonrelativistic limits. Later, the same result is obtained in [20] for $P(\lambda)=\left(\lambda+m^{2}\right)^{s / 2}-$ $\mathrm{m}^{s}$. We note that this family of solutions cannot be come from ground states or any variationally constructed solutions because the energy space controlled by the pseudo relativistic symbol $\left(\lambda+m^{2}\right)^{s / 2}-m^{s}$ is $H^{s / 2}$. In the following theorem, we generalize these results to Eq. (1.4) with the symbol $P$ satisfying (H1)-(H4).

Theorem 1.4 (Existence of a radial solution for $H^{1}$ subcritical range of $p$ ). Suppose (H1)(H4). For any $p \in(1,(d+2) /(d-2))$ and any $q>d / s$, there exists $\mu_{0}>0$ such that if $\mu \in\left(0, \mu_{0}\right)$ then there exists a radially symmetric nontrivial solution $u \in W^{s, q}\left(\mathbb{R}^{d}\right)$ to (1.4).

The rest of this paper is organized as follows. In Section 2, we introduce the concept of Bernstein functions and the properties of Fourier multiplier operators made by Bernstein symbols. In Section 3, we construct a nontrivial radial solution to (1.4) for small mass $\mu>0$ by using perturbation argument. Section 4 is devoted to the study of existence and qualitative properties on ground states to (1.4).

\section{Preliminaries}

\subsection{Bernstein functions}

A continuous function $f:[0, \infty) \rightarrow[0, \infty)$ is said to be totally monotone if it is smooth on $(0, \infty)$, and

$$
(-1)^{n} f^{(n)}(\lambda) \geq 0
$$

for all nonnegative integer $n$ and $\lambda>0$, where $f^{(n)}$ is the $n$-th derivative of $f$. Totally monotone functions are an important class of functions in many areas of analysis. We refer the book [21] for a comprehensive overview. As for totally monotone functions, an important theorem is Bernstein's theorem. It asserts that a totally monotone function is the Laplace transform of a Borel measure.

Theorem 2.1 (Bernstein's theorem [2]). If $f:[0, \infty) \rightarrow[0, \infty)$ is totally monotone, then there exists a non-negative Borel measure $\mu$ on $[0, \infty)$ such that

$$
f(\lambda)=\int_{0}^{\infty} e^{-t \lambda} d \mu(t)
$$

From now on, we assume that $f:[0, \infty) \rightarrow[0, \infty)$ and $f^{\prime}$ is totally monotone. Such a function is called a Bernstein function. Note that the symbols $\left(\lambda+m^{2}\right)^{s}-m^{2 s}, 0<s<1$ 
for the pseudo-relativistic operator or $\lambda^{s}, 0<s<1$ for the fractional Laplacian are examples of Bernstein functions. We define the Hilbert space $H_{f}\left(\mathbb{R}^{d} ; \mathbb{R}\right)$ by the completion of $C_{c}^{\infty}\left(\mathbb{R}^{d} ; \mathbb{R}\right)$ with respect to the norm

$$
\|u\|_{H_{f}}:=\left\{\int_{\mathbb{R}^{d}} f(-\Delta) u \cdot u d x\right\}^{1 / 2} .
$$

This function space is equipped with the inner product

$$
\langle u, v\rangle_{H_{f}}:=\int_{\mathbb{R}^{d}} f(-\Delta) u \cdot v d x .
$$

First, we prove that the symmetric decreasing rearrangement reduces the norm.

Proposition 2.1 (Pólya-Szegö inequality). Suppose that $f:[0, \infty) \rightarrow[0, \infty)$ is a Bernstein function. Then, for any non-negative function $u \in H_{f}\left(\mathbb{R}^{d} ; \mathbb{R}\right)$, we have

$$
\left\|u^{*}\right\|_{H_{f}\left(\mathbb{R}^{d} ; \mathbb{R}\right)} \leq\|u\|_{H_{f}\left(\mathbb{R}^{d} ; \mathbb{R}\right)},
$$

where $u^{*}$ is the symmetric decreasing rearrangement of $u$.

Proof. We claim that if $f$ is a Bernstein function, then $e^{-f(\lambda)}$ is totally monotone. For the claim, it suffices to show that the $n$-th derivative of $e^{-f(\lambda)}$ is of the form $(-1)^{n} e^{-f(\lambda)} g_{n}(\lambda)$, where $g_{n}$ is a (non-negative) totally monotone function. When $n=0$, it is obviously true with $g_{0}=1$. Suppose that the claim holds when $n=k$. Then, the next order derivative of $e^{-f(\lambda)}$ is $(-1)^{k+1} e^{-f(\lambda)} g_{k+1}(\lambda)$, where

$$
g_{k+1}(\lambda):=f^{\prime}(\lambda) g_{k}(\lambda)-g_{k}^{\prime}(\lambda),
$$

and $g_{k+1}(t)$ satisfies

$$
(-1)^{n} g_{k+1}^{(n)}(\lambda)=(-1)^{n}\left\{\sum_{\ell=0}^{n} f^{(\ell+1)}(\lambda) g_{k}^{(n-\ell)}(\lambda)-g_{k}^{(n+1)}(\lambda)\right\} \geq 0 .
$$

By functional calculus, we write

$$
\begin{aligned}
& \|u\|_{H_{f}\left(\mathbb{R}^{d} ; \mathbb{R}\right)}^{2}=\langle f(-\Delta) u, u\rangle_{L^{2}\left(\mathbb{R}^{d} ; \mathbb{R}\right)} \\
= & -\left.\frac{d}{d s}\right|_{s=0^{+}}\left\langle e^{-s f(-\Delta)} u, u\right\rangle_{L^{2}\left(\mathbb{R}^{d} ; \mathbb{R}\right)} \\
= & \lim _{s \rightarrow 0+} \frac{1}{s}\left\{\|u\|_{L^{2}\left(\mathbb{R}^{d} ; \mathbb{R}\right)}^{2}-\left\langle e^{-s f(-\Delta)} u, u\right\rangle_{L^{2}\left(\mathbb{R}^{d} ; \mathbb{R}\right)}\right\} .
\end{aligned}
$$

Note that by the claim and Bernstein's theorem, for each $s \geq 0$, there exists a non-negative Borel measure $\mu_{s}$ such that

$$
e^{-s f(\lambda)}=\int_{0}^{\infty} e^{-t \lambda} d \mu_{s}(t)
$$


Hence, inserting $\lambda=-\Delta$, we see that the operator $e^{-s f(-\Delta)}$ is the convolution of the radially symmetric, non-negative and decreasing function

$$
K_{s}(x)=\int_{0}^{\infty} \frac{1}{(4 \pi t)^{d / 2}} e^{-\frac{|x|^{2}}{4 t}} d \mu_{s}(t) .
$$

Therefore, by the Riesz rearrangement inequality, we conclude that

$$
\begin{aligned}
\|u\|_{H_{f}\left(\mathbb{R}^{d} ; \mathbb{R}\right)}^{2} & =\lim _{s \rightarrow 0+} \frac{1}{s}\left\{\|u\|_{L^{2}\left(\mathbb{R}^{d} ; \mathbb{R}\right)}^{2}-\iint_{\mathbb{R}^{d} \times \mathbb{R}^{d}} K_{s}(x-y) u(x) u(y) d x d y\right\} \\
& \geq \lim _{s \rightarrow 0+} \frac{1}{s}\left\{\left\|u^{*}\right\|_{L^{2}\left(\mathbb{R}^{d} ; \mathbb{R}\right)}^{2}-\iint_{\mathbb{R}^{d} \times \mathbb{R}^{d}} K_{s}(x-y) u^{*}(x) u^{*}(y) d x d y\right\}=\cdots \\
& =\left\|u^{*}\right\|_{H_{f}\left(\mathbb{R}^{d} ; \mathbb{R}\right)^{2}}^{2}
\end{aligned}
$$

Thus we complete the proof.

Next, we prove the symmetry and the positivity of the fundamental solution.

Proposition 2.2 (Positivity of the fundamental solution). If $f:[0, \infty) \rightarrow[0, \infty)$ is a Bernstein function, then the fundamental solution $\Phi=\Phi_{f}$ for the differential operator $f(-\Delta)$ is radially symmetric, strictly positive and decreasing.

Proof. It follows from

$$
f(-\Delta)^{-1}=\int_{0}^{\infty} e^{-s f(-\Delta)} d s
$$

(2.1) and the heat kernel.

We also show that a real-valued function in $H_{f}$ can be orthogonally decomposed into two functions having different signs.

Proposition 2.3 (Orthogonal decomposition). Suppose that $f:[0, \infty) \rightarrow[0, \infty)$ is a Bernstein function. Then, for any $u \in H_{f}\left(\mathbb{R}^{d} ; \mathbb{R}\right)$, there exist $u_{ \pm} \in H_{f}\left(\mathbb{R}^{d} ; \mathbb{R}\right)$ such that $u=u_{+}-u_{-}, u_{ \pm} \geq 0$ a.e. and $\left\langle u_{+}, u_{-}\right\rangle_{H_{f}\left(\mathbb{R}^{d} ; \mathbb{R}\right)}=0$.

Proof. Let $\mathcal{K}$ be the set of non-negative functions in $H_{f}\left(\mathbb{R}^{d} ; \mathbb{R}\right)$, which is a closed convex non-empty cone in $H_{f}\left(\mathbb{R}^{d} ; \mathbb{R}\right)$. Then, by Theorem 3.4 in [12] (see [19] for the original work), there exists a unique decomposition $u=u_{1}+u_{2}$, with $u_{1} \in \mathcal{K}$ and $u_{2} \in \mathcal{K}^{*}$, such that $\left\langle u_{1}, u_{2}\right\rangle_{H_{f}\left(\mathbb{R}^{d} ; \mathbb{R}\right)}=0$, where $\mathcal{K}^{*}$ is the dual cone of $\mathcal{K}$ defined by

$$
\mathcal{K}^{*}:=\left\{w \in H_{f}\left(\mathbb{R}^{d} ; \mathbb{R}\right):\langle w, u\rangle_{H_{f}} \leq 0 \text { for all } u \in \mathcal{K}\right\} .
$$

It remains to show that every $w \in \mathcal{K}^{*}$ is non-positive. Indeed, if $u \in C_{c}^{\infty}\left(\mathbb{R}^{d} ; \mathbb{R}\right)$ is nonnegative, then by Proposition 2.2, so is $\Phi * u$. Therefore, for any $w \in \mathcal{K}^{*}$, we have

$$
\langle w, u\rangle_{L^{2}\left(\mathbb{R}^{d} ; \mathbb{R}\right)}=\langle f(-\Delta) w, \Phi * u\rangle_{L^{2}\left(\mathbb{R}^{d} ; \mathbb{R}\right)}=\langle w, \Phi * u\rangle_{H_{f}\left(\mathbb{R}^{d} ; \mathbb{R}\right)} \leq 0 .
$$

However, since $u$ is arbitrary, this proves that $w$ is non-positive. 


\subsection{Assumptions on the symbol $P$ and its properties}

Here we recall the structural assumptions for the symbol $P$ :

(H1) $P:[0, \infty) \rightarrow[0, \infty)$ is continuous on $[0, \infty)$ and smooth on $(0, \infty)$;

(H2) $P$ is a Bernstein function, i.e., $P^{\prime}$ is totally monotone;

(H3) there exists $s \in(0,2]$ such that $P(\lambda) \gtrsim \lambda^{\frac{s}{2}}$ for all large $\lambda$;

(H4) $P(0)=0, P^{\prime}(0)=1$ and $P^{\prime \prime}(0)$ exists.

The advantage of assuming ( $\mathrm{H} 2)$ is to provide a nice integral representation of the symbol from which several important properties in our analysis are deduced.

Lemma 2.1 (Integral representation of the symbol). Suppose that the symbol $P:[0, \infty) \rightarrow \mathbb{R}$ satisfies (H2). Then, there exists a unique measure $\mu$ on $[0, \infty)$ such that

$$
P(\lambda)=\lambda \int_{0}^{1} \int_{0}^{\infty} e^{-\lambda \ell t} d \mu(t) d \ell=\int_{0}^{\infty} \frac{1-e^{-\lambda t}}{t} d \mu(t)
$$

As a consequence, if we further assume (H4), then the zeroth and the first moments of the measure is finite and

$$
\int_{0}^{\infty} t^{k} d \mu(t)=(-1)^{k} P^{(k+1)}(0), \quad k=0,1 .
$$

Proof. By the fundamental theorem of calculus, we have

$$
P(\lambda)=\int_{0}^{1} \frac{d}{d \ell}(P(\lambda \ell)) d \ell=\lambda \int_{0}^{1} P^{\prime}(\lambda \ell) d \ell .
$$

By (H2), it follows from Bernstein's theorem for complete monotone functions [21, Theorem 1.4] that

$$
P^{\prime}(\lambda)=\int_{0}^{\infty} e^{-\lambda t} d \mu(t)
$$

for some unique measure $\mu$ on $[0, \infty)$. Therefore, inserting the integral formula for $P^{\prime}(\lambda)$ and then integrating in $\ell$, we obtain (2.2). bol.

By $(\mathrm{H} 2)$, the symbol $P$ is sub-linear, and differentiation reduces the degree of the sym-

Lemma 2.2 (Properties of the symbol). Suppose that $P:[0, \infty) \rightarrow \mathbb{R}$ satisfies (H2).

(i) For all $\lambda_{1}, \lambda_{2} \geq 0$, we have $P\left(\lambda_{1}+\lambda_{2}\right) \leq P\left(\lambda_{1}\right)+P\left(\lambda_{2}\right)$.

(ii) For any integer $k \geq 0$ and $\lambda \geq 0$, we have $\left|P^{(k)}(\lambda)\right| \lesssim \lambda^{-k} P(\lambda)$. 
Proof. (i) By (2.2), we have

$$
\begin{aligned}
P\left(\lambda_{1}+\lambda_{2}\right) & =\left\{\int_{0}^{1} \int_{0}^{\infty} e^{-\left(\lambda_{1}+\lambda_{2}\right) \ell t} d \mu(t) d \ell\right\}\left(\lambda_{1}+\lambda_{2}\right) \\
& \leq\left\{\int_{0}^{1} \int_{0}^{\infty} e^{-\lambda_{1} \ell t} d \mu(t) d \ell\right\} \lambda_{1}+\left\{\int_{0}^{1} \int_{0}^{\infty} e^{-\lambda_{2} \ell t} d \mu(t) d \ell\right\} \lambda_{2} \\
& =P\left(\lambda_{1}\right)+P\left(\lambda_{2}\right) .
\end{aligned}
$$

(ii) Differentiating (2.2) $k$ times, we write

$$
\left|\lambda^{k} P^{(k)}(\lambda)\right|=\int_{0}^{\infty} \frac{(\lambda t)^{k} e^{-\lambda t}}{t} d \mu(t)
$$

and then apply the elementary inequality

$$
(\lambda t)^{k} e^{-\lambda t} \lesssim 1 \lesssim 1-e^{-\lambda t}
$$

Thus we complete the proof.

\section{Construction of a radial solution to the generalized NLS in $H^{1}$ subcritical range of $p$}

This section is devoted to prove Theorem 1.4. Consider a one-parameter family of nonlinear elliptic equations

$$
P_{\epsilon}(-\Delta) u+\mu u=|u|^{p-1} u,
$$

where $P_{\epsilon}(\lambda)=P(\epsilon \lambda) / \lambda$. If $P^{\prime}(0)$ exists, Eq. (3.1) formally converges to the nonlinear elliptic equation

$$
-\Delta u+\mu u=|u|^{p-1} u
$$

as $\epsilon \rightarrow 0$. To prove Theorem 1.4, we may construct a nontrivial radial solution to (3.1) for small $\epsilon>0$ since (3.1) is equivalent to (1.4) by the scaling $u(x)=\epsilon^{\frac{1}{p-1}} v(\epsilon x)$.

We assume that the nonlinearity is $H^{1}$-subcritical, i.e.,

$$
\max \left\{\frac{d-2}{d+2}, 0\right\}<\frac{1}{p}<1 .
$$

We remark that Eq. (3.1) is possibly supercritical, since the differential operator may have a lower order than the Laplacian (see Lemma 2.2(i)).

In this section, we impose the hypotheses $(\mathrm{H} 1)-(\mathrm{H} 4)$ on the symbol $P$ to establish existence of a non-trivial solution to (3.1) by the contraction mapping argument in [6] provided that $\epsilon$ is sufficiently small.

The following two lemmas will be employed in the contraction mapping argument. The first lemma asserts a certain coercivity of the Fourier multiplier $P_{\epsilon}(-\Delta)$. 
Lemma 3.1 (Coercivity). Suppose that (H1)-(H3) hold for some $s \in(0,2]$. Let $\mu>0$. Then, for any $1<q<\infty$, we have

$$
\left\|\frac{1}{P_{\epsilon}(-\Delta)+\mu}\right\|_{\mathcal{L}\left(L^{q ;} ; W^{s, q}\right)} \lesssim 1
$$

where $\|\cdot\|_{\mathcal{L}\left(L^{q} ; W^{s, q}\right)}$ denotes the standard operator norm from $L^{q}$ to $W^{s, q}$.

Proof. By the Hörmander-Mikhlin theorem, it suffices to show that for all integer $k \geq 0$,

$$
\left|\left(\frac{d}{d \lambda}\right)^{k}\left(\frac{1}{P_{\epsilon}(\lambda)+\mu}\right)\right| \lesssim \frac{1}{\lambda^{k}(\lambda+\mu)^{\frac{s}{2}}} .
$$

First, we claim that the $k$-th derivative of $\frac{1}{P_{\epsilon}(\lambda)+\mu}$ is the linear combination of products of fractions of the form

$$
\frac{1}{P_{\epsilon}(\lambda)+\mu} \cdots \frac{P_{\epsilon}^{\left(j_{1}\right)}(\lambda)}{P_{\epsilon}(\lambda)+\mu} \cdots \frac{P_{\epsilon}^{\left(j_{m}\right)}(\lambda)}{P_{\epsilon}(\lambda)+\mu}
$$

with $j_{1}+\cdots j_{m}=k$. We prove the claim by induction. The zeroth step is trivial. If the claim is true for the $k$-th step, then the $(k+1)$-th step follows, because when the derivative hits one of the fractions, it generates fractions of the same kind. Precisely, we have

$$
\left(\frac{P_{\epsilon}^{(j)}(\lambda)}{P_{\epsilon}(\lambda)+\mu}\right)^{\prime}=\frac{P_{\epsilon}^{(j+1)}(\lambda)}{P_{\epsilon}(\lambda)+\mu}-\frac{P_{\epsilon}^{(j)}(\lambda)}{P_{\epsilon}(\lambda)+\mu} \cdot \frac{P_{\epsilon}^{\prime}(\lambda)}{P_{\epsilon}(\lambda)+\mu} .
$$

Now, we show (3.4) by induction again. The zeroth step follows from the assumption (H3). Suppose that (3.4) holds for $k$. Then, each term in the $k$-th derivative (see (3.5)) is bounded by $\lambda^{-k}(\lambda+\mu)^{-\frac{s}{2}}$. However, when the derivative hits one of the factor (like (3.6)), one can see from Lemma 2.2(ii) that an extra factor $\lambda^{-1}$ is gained.

The second lemma claims the convergence $P_{\epsilon}(-\Delta) \rightarrow-\Delta$ in the norm resolvent sense.

Lemma 3.2 (Norm resolvent convergence of $\left.P_{\epsilon}\right)$. Suppose that $(\mathrm{H} 1)-(\mathrm{H} 4)$ hold for some $s \in$ $(0,2]$. Let $\mu>0$. Then, for any $1<q<\infty$, we have

$$
\left\|\frac{1}{P_{\epsilon}(-\Delta)+\mu}-\frac{1}{-\Delta+\mu}\right\|_{\mathcal{L}\left(L^{q} ; L^{q}\right)} \lesssim \epsilon^{\frac{s}{2}} .
$$

Proof. By the Hörmander-Mikhlin theorem again, it suffices to show that

$$
\left|\left(\frac{d}{d \lambda}\right)^{k}\left(\frac{1}{P_{\epsilon}(\lambda)+\mu}-\frac{1}{\lambda+\mu}\right)\right| \lesssim \frac{\epsilon^{\frac{s}{2}}}{\lambda^{k}} .
$$


If $\lambda \geq \frac{1}{\epsilon}$, then we have

$$
\left|\left(\frac{d}{d \lambda}\right)^{k}\left(\frac{1}{\lambda+\mu}\right)\right| \lesssim(\lambda+\mu)^{-(k+1)} \lesssim \epsilon \lambda^{-k}
$$

and by (3.4), we have

$$
\left|\left(\frac{d}{d \lambda}\right)^{k}\left(\frac{1}{P_{\epsilon}(\lambda)+\mu}\right)\right| \lesssim \lambda^{-k-\frac{s}{2}} \lesssim \epsilon^{\frac{s}{2}} \lambda^{-k}
$$

Suppose that $\lambda \leq \frac{1}{\epsilon}$. In this case, we enjoy cancelation from the difference. We write

$$
\frac{1}{P_{\epsilon}(\lambda)+\mu}-\frac{1}{\lambda+\mu}=\frac{\lambda-P_{\epsilon}(\lambda)}{\left(P_{\epsilon}(\lambda)+\mu\right)(\lambda+\mu)}=\frac{(\lambda+\mu)^{\frac{s}{2}}}{P_{\epsilon}(\lambda)+\mu} \frac{1}{(\lambda+\mu)^{1+\frac{s}{2}}}\left(\lambda-P_{\epsilon}(\lambda)\right) .
$$

It is obvious that

$$
\left|\left(\frac{d}{d \lambda}\right)^{k}(\lambda+\mu)^{-1-\frac{s}{2}}\right| \lesssim \lambda^{-k-1-\frac{s}{2}}
$$

and we have shown that

$$
\left|\left(\frac{d}{d \lambda}\right)^{k}\left(\frac{(\lambda+\mu)^{\frac{s}{2}}}{P_{\epsilon}(\lambda)+\mu}\right)\right| \lesssim \lambda^{-k}
$$

(see (3.4)). Thus, it remains to consider the last factor $\left(\lambda-P_{\epsilon}(\lambda)\right)$. Indeed, differentiating (2.2) and using (2.3), one can show that

$$
\begin{aligned}
& \left|\lambda-P_{\epsilon}(\lambda)\right| \leq \int_{0}^{\infty}\left|\frac{1-\epsilon \lambda t-e^{-\epsilon \lambda t}}{\epsilon t}\right| d \mu(t) \leq \frac{1}{2} \int_{0}^{\infty} \epsilon t \lambda^{2} d \mu(t)=\frac{\epsilon \lambda^{2}\left|P^{\prime \prime}(0)\right|}{2} \lesssim \epsilon^{\frac{s}{2}} \lambda^{1+\frac{s}{2}}, \\
& \left|\left(\lambda-P_{\epsilon}(\lambda)\right)^{\prime}\right|=\int_{0}^{\infty} 1-e^{-\epsilon \lambda t} d \mu(t) \leq \int_{0}^{\infty}(\epsilon t \lambda) d \mu(t)=\epsilon \lambda P^{\prime}(0) \leq \epsilon^{\frac{s}{2}} \lambda^{\frac{s}{2}},
\end{aligned}
$$

and that for $k \geq 2$,

$$
\begin{aligned}
\left|\left(\lambda-P_{\epsilon}(\lambda)\right)^{(k)}\right| & =\int_{0}^{\infty}(\epsilon t)^{k-1} e^{-\epsilon \lambda t} d \mu(t) \lesssim \int_{0}^{\infty}(\epsilon t) \lambda^{-k+2} d \mu(t) \\
& =\epsilon \lambda^{-k+2} P^{\prime}(0) \leq \epsilon^{\frac{s}{2}} \lambda^{1+\frac{s}{2}-k}
\end{aligned}
$$

Therefore, collecting all, we complete the proof of (3.7).

\subsection{Construction of a non-trivial solution}

Now, we seek for a solution of the form near the ground state to the limit equation. Note that Theorem 3.1 below obviously includes Theorem 1.4.

Theorem 3.1 (Existence and local uniqueness of a non-trivial solution). Suppose that (H1)(H4) for some $s \in(0,2]$. Assuming (3.3), let $u_{0}$ be the unique radially symmetric solution to the limit equation (3.2). Then, there exists small $\epsilon_{0}>0$ such that $E q$. (3.1) has a non-trivial radially symmetric solution $u_{\epsilon} \in W^{s, q}$ for all $r \geq 2$. Moreover, $u_{\epsilon}$ is a unique radial solution in the neighborhood of $u_{0}$ with respect to the norm $\|\cdot\|_{W^{s, q} \text {. }}$ 
We insert $u_{\epsilon}=u_{0}+w_{\epsilon}$, assuming that the difference $w_{\epsilon}$ is small. Then, reorganizing in the linearized form, the equation for $w_{\epsilon}$ is derived as

$$
\begin{aligned}
& \left(P_{\epsilon}(-\Delta)+\mu-p u_{0}^{p-1}\right) w_{\epsilon} \\
= & \left(-\Delta-P_{\epsilon}(-\Delta)\right) u_{0}+\left\{\left(u+w_{\epsilon}\right)^{p}-u_{0}^{p}-p u_{0}^{p-1} w_{\epsilon}\right\} .
\end{aligned}
$$

We claim that the linearized operator on the left hand side is invertible.

Lemma 3.3 (Invertibility of the linearized operator). Suppose that (H1)-(H4) hold for some $s \in(0,2]$. Then, for any $q \in[2, \infty)$, there exists small $\epsilon_{0}>0$ such that if $0<\epsilon \leq \epsilon_{0}$, then the operator

$$
P_{\epsilon}(-\Delta)+\mu-p u_{0}^{p-1}: W_{r}^{s, q} \rightarrow L_{r}^{q}
$$

is invertible, where $L_{r}^{q}$ and $W_{r}^{s, q}$ are the radial sub-spaces of $L^{q}$ and $W^{s, q}$ respectively. Moreover, we have

$$
\sup _{0<\epsilon \leq \epsilon_{0}}\left\|\left(P_{\epsilon}(-\Delta)+\mu-p u_{0}^{p-1}\right)^{-1}\right\|_{\mathcal{L}\left(L_{r}^{q} ; W_{r}^{s, q}\right)}<\infty
$$

Proof. We write

$$
\begin{aligned}
& P_{\epsilon}(-\Delta)+\mu-p u_{0}^{p-1} \\
= & \left(\operatorname{Id}-p u_{0}^{p-1}\left(P_{\epsilon}(-\Delta)+\mu\right)^{-1}\right)\left(P_{\epsilon}(-\Delta)+\mu\right) \\
= & \left(\mathcal{A}+p u_{0}^{p-1}\left(\frac{1}{-\Delta+\mu}-\frac{1}{P_{\epsilon}(-\Delta)+\mu}\right)\right)\left(P_{\epsilon}(-\Delta)+\mu\right),
\end{aligned}
$$

where

$$
\mathcal{A}=\mathrm{Id}-p u_{0}^{p-1}(-\Delta+\mu)^{-1} .
$$

Using invertibility of the operator $\mathcal{A}: L_{r}^{q} \rightarrow L_{r}^{q}$ for all $q \geq 2$ (see [6, Lemma 3.2]), we factor out $\mathcal{A}$ to the right,

$$
\begin{aligned}
& P_{\epsilon}(-\Delta)+\mu-p u_{0}^{p-1} \\
= & \left\{\operatorname{Id}+p u_{0}^{p-1}\left(\frac{1}{-\Delta+\mu}-\frac{1}{P_{\epsilon}(-\Delta)+\mu}\right) \mathcal{A}^{-1}\right\} \mathcal{A}\left(P_{\epsilon}(-\Delta)+\mu\right) .
\end{aligned}
$$

Since the ground state $u_{0}$ is bounded, it follows from Lemma 3.2 that for small $0<\epsilon \leq \epsilon_{0}$,

$$
\begin{gathered}
\left\|p u_{0}^{p-1}\left(\frac{1}{-\Delta+\mu}-\frac{1}{P_{\epsilon}(-\Delta)+\mu}\right) \mathcal{A}^{-1}\right\|_{\mathcal{L}\left(L_{r}^{q} \rightarrow L_{r}^{q}\right)} \\
\leq p\left\|u_{0}\right\|_{L^{\infty}}^{p-1}\left\|\frac{1}{-\Delta+\mu}-\frac{1}{P_{\epsilon}(-\Delta)+\mu}\right\|_{\mathcal{L}\left(L_{r}^{q} \rightarrow L_{r}^{q}\right)}\left\|\mathcal{A}^{-1}\right\|_{\mathcal{L}\left(L_{r}^{q} \rightarrow L_{r}^{q}\right)} \leq \frac{1}{2} .
\end{gathered}
$$


Therefore,

$$
\mathrm{Id}+p u_{0}^{p-1}\left(\frac{1}{-\Delta+\mu}-\frac{1}{P_{\epsilon}(-\Delta)+\mu}\right) \mathcal{A}^{-1}
$$

is invertible on $L_{r}^{q}$, and its inverse is uniformly bounded. Therefore, inverting (3.9) and applying Lemma 3.1, we complete the proof.

By the above lemma, Eq. (3.8) can be written as

$$
\begin{aligned}
w_{\epsilon}=( & \left.P_{\epsilon}(-\Delta)+\mu-p u_{0}^{p-1}\right)^{-1}\left(-\Delta-P_{\epsilon}(-\Delta)\right) u_{0} \\
& +\left(P_{\epsilon}(-\Delta)+\mu-p u_{0}^{p-1}\right)^{-1}\left\{\left(u_{0}+w_{\epsilon}\right)^{p}-u_{0}^{p}-p u_{0}^{p-1} w_{\epsilon}\right\} .
\end{aligned}
$$

We aim to find a solution to (3.10) by a contraction mapping argument. The following elementary inequality is helpful to handle the nonlinearity in the equation.

Lemma 3.4. If $a>0$ and $p>1$, then

$$
\begin{aligned}
& || a+\left.b\right|^{p-1}(a+b)-|a+\tilde{b}|^{p-1}(a+\tilde{b})-p a^{p-1}(b-\tilde{b}) \mid \\
\lesssim & \begin{cases}(|b|+|\tilde{b}|)^{p-1}|b-\tilde{b}|, & \text { if } 1<p \leq 2, \\
(a+|b|+|\tilde{b}|)^{p-2}(|b|+|\tilde{b}|)|b-\tilde{b}|, & \text { if } p \geq 2 .\end{cases}
\end{aligned}
$$

Proof. By the fundamental theorem of calculus,

$$
\begin{aligned}
& |a+b|^{p-1}(a+b)-|a+\tilde{b}|^{p-1}(a+\tilde{b})-p a^{p-1}(b-\tilde{b}) \\
= & p(b-\tilde{b}) \int_{0}^{1}\left\{|a+(b-\tilde{b}) \ell|^{p-1}-a^{p-1}\right\} d \ell .
\end{aligned}
$$

If $1<p \leq 2$, using the inequality

$$
\left.|| \alpha\right|^{p-1}-|\beta|^{p-1}|\leq||\alpha|-\left.|\beta|\right|^{p-1} \leq|\alpha-\beta|^{p-1},
$$

we obtain the desired bound. If $p>2$, we apply the fundamental theorem of calculus again to get

$$
\begin{aligned}
& |a+b|^{p-1}(a+b)-|a+\tilde{b}|^{p-1}(a+\tilde{b})-p a^{p-1}(b-\tilde{b}) \\
= & p(p-1)(b-\tilde{b})^{2} \int_{0}^{1} \int_{0}^{\ell}\left\{\left|a+(b-\tilde{b}) \ell^{\prime}\right|^{p-3}\left(a+(b-\tilde{b}) \ell^{\prime}\right)-a^{p-2}\right\} d \ell^{\prime} d \ell .
\end{aligned}
$$

Then, one can show the bound.

Proof of Theorem 3.1. We define

$$
\begin{aligned}
\Phi(w)=( & \left.P_{\epsilon}(-\Delta)+\mu-p u_{0}^{p-1}\right)^{-1}\left(-\Delta-P_{\epsilon}(-\Delta)\right) u_{0} \\
& +\left(P_{\epsilon}(-\Delta)+\mu-p u_{0}^{p-1}\right)^{-1}\left\{\left(u_{0}+w\right)^{p}-u_{0}^{p}-p u_{0}^{p-1} w\right\} .
\end{aligned}
$$


For sufficiently small $\delta>0$ to be chosen later, we denote

$$
B_{\delta}(0)=\left\{w:\|w\|_{H_{r}^{s} \cap W_{r}^{s, q}} \leq \delta\right\}
$$

for some $q>\frac{d}{s}$. Then, by Lemma 3.3, we obtain

$$
\begin{gathered}
\|\Phi(w)\|_{H_{r}^{s} \cap W_{r}^{s, q}} \lesssim\left\|\left(-\Delta-P_{\epsilon}(-\Delta)\right) u_{0}\right\|_{L_{r}^{2} \cap L_{r}^{q}}+\left\|\left(u_{0}+w\right)^{p}-u_{0}^{p}-p u_{0}^{p-1} w\right\|_{L_{r}^{2} \cap L_{r}^{q}}, \\
\left\|\Phi\left(w_{1}\right)-\Phi\left(w_{2}\right)\right\|_{H_{r}^{s} \cap W_{r}^{s, q}} \lesssim \|\left\{\left(u_{0}+w_{1}\right)^{p}-u_{0}^{p}-p u_{0}^{p-1} w_{1}\right\} \\
-\left\{\left(u_{0}+w_{2}\right)^{p}-u_{0}^{p}-p u_{0}^{p-1} w_{2}\right\} \|_{L_{r}^{2} \cap L_{r}^{q}} .
\end{gathered}
$$

For $\left(-\Delta-P_{\epsilon}(-\Delta)\right) u_{0}$, we recall that $u_{\infty} \in H_{r}^{2} \cap W_{r}^{2, q}$ for all $q \geq 2$. Therefore, by density, there exists $u_{0, \delta^{2}} \in C_{c}^{\infty}$ such that $\left\|u_{0}-u_{0, \delta^{2}}\right\|_{H_{r}^{2} \cap W_{r}^{2, q}} \leq \delta^{2}$. Then, we have

$$
\begin{aligned}
& \left\|\left(-\Delta-P_{\epsilon}(-\Delta)\right)\left(u_{0}-u_{0, \delta^{2}}\right)\right\|_{L_{r}^{2} \cap L_{r}^{q}} \lesssim\left\|u_{0}-u_{0, \delta^{2}}\right\|_{H_{r}^{2} \cap W_{r}^{2, q}} \leq \delta^{2}, \\
& \left\|\left(-\Delta-P_{\epsilon}(-\Delta)\right) u_{0, \delta^{2}}\right\|_{L_{r}^{2} \cap L_{r}^{q}} \rightarrow 0 \quad \text { as } \epsilon \rightarrow 0 .
\end{aligned}
$$

Therefore, there exists small $\epsilon_{0}>0$ (depending on the function $u_{0}$ ) such that for $\epsilon \in$ $\left(0, \epsilon_{0}\right]$,

$$
\left\|\left(-\Delta-P_{\epsilon}(-\Delta)\right) u_{0}\right\|_{L_{r}^{2} \cap L_{r}^{q}} \leq \frac{\delta}{2} \text {. }
$$

For the nonlinear terms, we apply Lemma 3.4 and Hölder's inequality and the Sobolev embedding $W^{s, q} \hookrightarrow L^{\infty}$ with $q>\frac{d}{s}$. Indeed, if $1<p \leq 2$, then

$$
\begin{aligned}
& \left\|\left(u_{0}+w\right)^{p}-u_{0}^{p}-p u_{0}^{p-1} w\right\|_{L_{r}^{2} \cap L_{r}^{q}} \\
\lesssim & \left\|\left.w\right|^{p-1}|w|\right\|_{L_{r}^{2} \cap L_{r}^{q}} \leq\|w\|_{L_{r}^{\infty}}^{p-1}\|w\|_{L_{r}^{2} \cap L_{r}^{q}} \lesssim \delta^{p-1}\|w\|_{L_{r}^{2} \cap L_{r}^{q},}
\end{aligned}
$$

while if $p>2$,

$$
\begin{aligned}
& \left\|\left(u_{0}+w\right)^{p}-u_{0}^{p}-p u_{0}^{p-1} w\right\|_{L_{r}^{2} \cap L_{r}^{q}} \\
\lesssim & \left\|\left(u_{0}+|w|\right)^{p-2}|w|^{2}\right\|_{L_{r}^{2} \cap L_{r}^{q}} \\
\lesssim & \left\{\left\|u_{\infty}\right\|_{L_{r}^{\infty}}+\|w\|_{L_{r}^{\infty}}\right\}^{p-2}\|w\|_{L_{r}^{2} \cap L_{r}^{q}}^{2} \leq \delta\|w\|_{L_{r}^{2} \cap L_{r}^{q}} .
\end{aligned}
$$

Similarly, one can show that

$$
\begin{aligned}
& \left\|\left\{\left(u_{0}+w_{1}\right)^{p}-u_{0}^{p}-p u_{0}^{p-1} w_{1}\right\}-\left\{\left(u_{0}+w_{2}\right)^{p}-u_{0}^{p}-p u_{0}^{p-1} w_{2}\right\}\right\|_{L_{r}^{2} \cap L_{r}^{q}} \\
\lesssim & \begin{cases}\delta^{p-1}\left\|w_{1}-w_{2}\right\|_{L_{r}^{2} \cap L_{r}^{q},} & \text { if } 1<p \leq 2, \\
\delta\left\|w_{1}-w_{2}\right\|_{L_{r}^{2} \cap L_{r}^{q},} & \text { if } p \geq 2 .\end{cases}
\end{aligned}
$$

Collecting all, we conclude that $\Phi$ is contractive on $B_{\delta}(0)$. Therefore, Eq. (3.10) (consequently, (3.1)) has a unique solution in $B_{\delta}(0)$. 


\section{Ground state for the generalized lower-order NLS}

In this section we prove Theorems 1.1-1.3. Throughout this section, $\mu$ denotes a positive constant.

\subsection{Variational settings}

For a symbol $P$ satisfying (H1)-(H3), we consider the Hilbert space $H_{P+\mu}=H_{P+\mu}\left(\mathbb{R}^{d} ; \mathbb{R}\right)$. We note that $P+\mu$ is a Bernstein function because so is $P$. By (P2) and Sobolev embedding, one has a natural embedding

$$
H_{P+\mu} \hookrightarrow H^{s}\left(\mathbb{R}^{d}\right) \hookrightarrow L^{2}\left(\mathbb{R}^{d}\right) \cap L^{2 d /(d-s)}\left(\mathbb{R}^{d}\right) .
$$

Then the functional $\mathcal{I}$ given by

$$
\mathcal{I}(u)=\frac{1}{2}\|u\|_{H_{P+\mu}}^{2}-\frac{1}{p+1} \int_{\mathbb{R}^{d}}|u|^{p+1} d x
$$

is well-defined and $C^{1}$ on $H_{P+\mu}$. It is clear that (1.4) is the Euler-Lagrange equation of (4.1).

We say $u_{0} \in H_{P+\mu}$ is a ground state to (1.4) if $u_{0}$ is a critical point of $\mathcal{I}$ and $\mathcal{I}\left(u_{0}\right) \leq$ $\mathcal{I}(v)$ for any nontrivial critical point $v \in H_{P+\mu}$ of $\mathcal{I}$.

\subsection{Existence of a ground state}

In this subsection, we construct a ground state for the generalized NLS, which is positive, radially symmetric and monotone decreasing up to translation. We shall be done by establishing existence of a minimizer for the variational problem

$$
\mathcal{I}_{\text {min }}=\inf _{0 \neq u \in H_{P+\mu}}\left\{\mathcal{I}(u):\left\langle\mathcal{I}^{\prime}(u), u\right\rangle_{L^{2}}=0\right\} .
$$

Such a minimizer is of course a ground state for the generalized NLS, since every critical point of $\mathcal{I}$ satisfies the constraint.

The following lemma is trivial, but we write it as a lemma, because it will be used frequently.

Lemma 4.1. Suppose that $u$ is admissible for the variational problem (4.2), i.e., $u \neq 0$ and

$$
\left\langle\mathcal{I}^{\prime}(u), u\right\rangle_{L^{2}}=\|u\|_{H_{P+\mu}}^{2}-\int_{\mathbb{R}^{d}}|u|^{p+1} d x=0 .
$$

We assume that

$$
\begin{aligned}
& \|\tilde{u}\|_{H_{P+\mu}} \leq\|u\|_{H_{P+\mu^{\prime}}} \\
& \int_{\mathbb{R}^{d}}|\tilde{u}|^{p+1} d x \geq \int_{\mathbb{R}^{d}}|u|^{p+1} d x .
\end{aligned}
$$


Then, there exists $t \in(0,1]$ such that $t \tilde{u}$ is admissible and

$$
\mathcal{I}(t \tilde{u}) \leq \mathcal{I}(u) .
$$

If we further assume that either the inequality (4.3a) or (4.3b) holds strictly, then

$$
\mathcal{I}(t \tilde{u})<\mathcal{I}(u) \text {. }
$$

Proof. Since

$$
\|\tilde{u}\|_{H_{P+\mu}}^{2}-\int_{\mathbb{R}^{d}}|\tilde{u}|^{p+1} d x \leq\|u\|_{H_{P+\mu}}^{2}-\int_{\mathbb{R}^{d}}|u|^{p+1} d x=0,
$$

there exists $t \in(0,1]$ such that $t \tilde{u}$ is admissible, i.e.,

$$
\|t \tilde{u}\|_{H_{P+\mu}}^{2}-\int_{\mathbb{R}^{d}}|t \tilde{u}|^{p+1} d x=t^{2}\left\{\|\tilde{u}\|_{H_{P+\mu}}^{2}-t^{p-1} \int_{\mathbb{R}^{d}}|\tilde{u}|^{p+1} d x\right\}=0 .
$$

Therefore, by (4.3a), it follows that

$$
\begin{aligned}
\mathcal{I}(t \tilde{u}) & =\frac{1}{2}\|t \tilde{u}\|_{H_{P+\mu}}^{2}-\frac{1}{p+1} \int_{\mathbb{R}^{d}}|t \tilde{u}|^{p+1} d x=\left(\frac{1}{2}-\frac{1}{p+1}\right)\|t \tilde{u}\|_{H_{P+\mu}}^{2} \\
& =\left(\frac{1}{2}-\frac{1}{p+1}\right) t^{2}\|\tilde{u}\|_{H_{P+\mu}}^{2} \leq\left(\frac{1}{2}-\frac{1}{p+1}\right)\|u\|_{H_{P+\mu}}^{2}=\mathcal{I}(u) .
\end{aligned}
$$

If either (4.3a) or (4.3b) holds strictly, then $t<1$ and $\mathcal{I}(t \tilde{u})<\mathcal{I}(u)$.

Proof of Theorem 1.1. By the constraint,

$$
\mathcal{I}(u)=\left(\frac{1}{2}-\frac{1}{p+1}\right)\|u\|_{H_{P+\mu}}^{2}
$$

for admissible $u$. Let $\left\{u_{n}\right\}_{n=1}^{\infty} \subset H_{P+\mu}$ be a minimizing sequence. For each $n$, applying Proposition 2.3, we write $u_{n}=u_{n,+}-u_{n,-}$ such that $u_{n, \pm} \in H_{P+\mu}, u_{ \pm} \geq 0$ and $\left\langle u_{n,+}, u_{n,-}\right\rangle_{H_{P+\mu}}=0$. We define a new sequence $\left\{\tilde{u}_{n}\right\}_{n=1}^{\infty}$ by $\tilde{u}_{n}=u_{n,+}+u_{n,-} \geq\left|u_{n}\right|$. Then, we have

$$
\left\|\tilde{u}_{n}\right\|_{H_{P+\mu}}^{2}=\left\|u_{n,+}\right\|_{H_{P+\mu}}^{2}+\left\|u_{n,-}\right\|_{H_{P+\mu}}^{2}=\left\|u_{n}\right\|_{H_{P+\mu}}^{2}
$$

but

$$
\begin{aligned}
& \int_{\mathbb{R}^{d}}\left|\tilde{u}_{n}\right|^{p+1} d x= \int_{\mathbb{R}^{d}}\left|u_{n,+}+u_{n,-}\right|^{p+1} d x \\
&= \int_{\operatorname{supp}\left(u_{n,+}\right) \backslash \operatorname{supp}\left(u_{n,-}\right)}\left|u_{n,+}\right|^{p+1} d x+\int_{\operatorname{supp}\left(u_{n,-}\right) \backslash \operatorname{supp}\left(u_{n,+}\right)}\left|u_{n,-}\right|^{p+1} d x \\
& \quad+\int_{\operatorname{supp}\left(u_{n,+}\right) \cap \operatorname{supp}\left(u_{n,-}\right)}\left|u_{n,+}+u_{n,-}\right|^{p+1} d x \\
& \geq \int_{\operatorname{supp}\left(u_{n,+}\right) \backslash \operatorname{supp}\left(u_{n,-}\right)}\left|u_{n,+}\right|^{p+1} d x+\int_{\operatorname{supp}\left(u_{n,-}\right) \backslash \operatorname{supp}\left(u_{n,+}\right)}\left|u_{n,-}\right|^{p+1} d x \\
& \quad+\int_{\operatorname{supp}\left(u_{n,+}\right) \cap \operatorname{supp}\left(u_{n,-}\right)}\left|u_{n,+}-u_{n,-}\right|^{p+1} d x \\
&=\int_{\mathbb{R}^{d}}\left|u_{n}\right|^{p+1} d x .
\end{aligned}
$$


Thus, it follows from Lemma 4.1 that there exists $\left\{t_{n}\right\}_{n=1}^{\infty} \subset(0,1]$ such that $\left\{t_{n} \tilde{u}_{n}\right\}_{n=1}^{\infty}$ is also a minimizing sequence. Replacing $u_{n}$ by $t_{n} \tilde{u}_{n}$, we assume that $u_{n}$ is nonnegative.

Let $u_{n}^{*}$, where $f^{*}$ is the symmetric decreasing rearrangements of $f \geq 0$. Then, by Pólya-Szegö inequality (Proposition 2.1) and the measure preserving property of the rearrangement,

$$
\left\|u_{n}^{*}\right\|_{H_{P+\mu}}^{2} \leq\left\|u_{n}\right\|_{H_{P+\mu^{\prime}}}^{2} \quad \int_{\mathbb{R}^{d}}\left|u_{n}^{*}\right|^{p+1} d x=\int_{\mathbb{R}^{d}}\left|u_{n}\right|^{p+1} d x .
$$

Hence, by Lemma 4.1 again, we can find a sequence $\left\{t_{n}\right\}_{n=1}^{\infty} \subset(0,1]$ such that $\left\{t_{n} u_{n}^{*}\right\}_{n=1}^{\infty}$ is also a minimizing sequence. Replacing $u_{n}$ by $t_{n} u_{n}^{*}$, we assume that $u_{n}$ is radially symmetric and monotone decreasing.

We now have a minimizing sequence $\left\{u_{n}\right\}_{n=1}^{\infty}$ of nonnegative radially symmetric functions monotone decreasing in the radial direction. Hence, passing to a subsequence, $\left\{u_{n}\right\}$ weakly converges to some $u$ in $H_{P+\mu}$, which implies that

$$
\|u\|_{H_{P+\mu}} \leq \liminf _{n \rightarrow \infty}\left\|u_{n}\right\|_{H_{P+\mu}} .
$$

On the other hand, by the compact embedding $H_{\text {rad }}^{s} \hookrightarrow L_{\text {rad }}^{q}$ with $2<q<2 d /(d-s)$, we have

$$
\lim _{n \rightarrow \infty} \int_{\mathbb{R}^{d}}\left|u_{n}\right|^{p+1} d x=\int_{\mathbb{R}^{d}}|u|^{p+1} d x
$$

and $\left\{u_{n}\right\}$ converges to $u$ a.e. This shows that $u$ is also nonnegative radial symmetric function monotone decreasing in radial direction.

We claim that $u \neq 0$. If not, $u_{n}$ strongly converges to 0 in $H_{P+\mu}$ since $\left\|u_{n}\right\|_{H_{P+\mu}}^{2}=$ $\left\|u_{n}\right\|_{p+1}^{p+1}$. However by Sobolev embedding, one has

$$
\left\|u_{n}\right\|_{H_{P+\mu}}^{2}=\left\|u_{n}\right\|_{p+1}^{p+1} \leq C\left\|u_{n}\right\|_{H_{P+\mu}}^{p+1} .
$$

This implies a positive lower bound of $\left\|u_{n}\right\|_{H_{P+\mu}}$, which makes a contradiction. Thus, choosing appropriate $t \in(0,1]$ by Lemma 4.1 as above, we can make $t u$ admissible, and

$$
\begin{aligned}
\mathcal{I}(t u) & =\left(\frac{1}{2}-\frac{1}{p+1}\right) t^{2}\|u\|_{H_{P+\mu}}^{2} \leq\left(\frac{1}{2}-\frac{1}{p+1}\right)\|u\|_{H_{P+\mu}}^{2} \\
& \leq \liminf _{n \rightarrow \infty}\left(\frac{1}{2}-\frac{1}{p+1}\right)\left\|u_{n}\right\|_{H_{P+\mu}}^{2}=\liminf _{n \rightarrow \infty} \mathcal{I}\left(u_{n}\right) .
\end{aligned}
$$

Therefore, we conclude that $t u$ is a minimizer.

We once more redefining $t u$ as $u$ so that $u$ is a nonnegative ground state to (1.4) which is radially symmetric and decreasing in radial direction. It remains to show that $u$ is strictly positive everywhere. Let $\Phi_{P+\mu}$ be the fundamental solution of the differential operator $P(-\Delta)+\mu$. By Proposition 2.2, it is strictly positive. Since the ground state $u$ is represented by

$$
u=\int_{\mathbb{R}^{d}} \Phi_{P+\mu}(x-y) u^{p}(y) d x,
$$

we see that $u$ is strictly positive. This completes the proof. 


\subsection{Sign-definiteness of a ground state}

This subsection is devoted to prove Theorem 1.2, the sign-definiteness of a ground state to (1.4).

Proof of Theorem 1.2. By Proposition 2.3, there are non-negative $u_{ \pm} \in H_{P+\mu}$ such that $u=$ $u_{+}-u_{-},\left\langle u_{+}, u_{-}\right\rangle_{H_{P+\mu}}=0$. We observe that $u_{+}(x) u_{-}(x)=0$ for all $x \in \mathbb{R}^{d}$. In other words, $u_{+}$and $u_{-}$have disjoint supports,

$$
\operatorname{supp}\left(u_{+}\right) \cap \operatorname{supp}\left(u_{-}\right)=\varnothing \text {. }
$$

Indeed, if (4.4) does not hold, the function $\tilde{u}=u_{+}+u_{-}$obeys $\|\tilde{u}\|_{H_{P+\mu}}^{2}=\|u\|_{H_{P+\mu}}^{2}$ and

$$
\begin{aligned}
\int_{\mathbb{R}^{d}}\left|\tilde{u}_{n}\right|^{p+1} d x= & \int_{\mathbb{R}^{d}}\left|u_{n,+}+u_{n,-}\right|^{p+1} d x \\
= & \int_{\operatorname{supp}\left(u_{n,+}\right) \backslash \operatorname{supp}\left(u_{n,-}\right)}\left|u_{n,+}\right|^{p+1} d x+\int_{\operatorname{supp}\left(u_{n,-}\right) \backslash \operatorname{supp}\left(u_{n,+}\right)}\left|u_{n,-}\right|^{p+1} d x \\
& \quad+\int_{\operatorname{supp}\left(u_{n,+}\right) \cap \operatorname{supp}\left(u_{n,-}\right)}\left|u_{n,+}+u_{n,-}\right|^{p+1} d x \\
> & \int_{\operatorname{supp}\left(u_{n,+}\right) \backslash \operatorname{supp}\left(u_{n,-}\right)}\left|u_{n,+}\right|^{p+1} d x+\int_{\operatorname{supp}\left(u_{n,-}\right) \backslash \operatorname{supp}\left(u_{n,+}\right)}\left|u_{n,-}\right|^{p+1} d x \\
& \quad+\int_{\operatorname{supp}\left(u_{n,+}\right) \cap \operatorname{supp}\left(u_{n,-}\right)}\left|u_{n,+}-u_{n,-}\right|^{p+1} d x \\
= & \int_{\mathbb{R}^{d}}\left|u_{n}\right|^{p+1} d x,
\end{aligned}
$$

where the inequality holds strictly due to cross terms. Thus, it follows from Lemma 4.1 that there exists $t \in(0,1]$ such that $t u$ is admissible and $\mathcal{I}(t u)<\mathcal{I}(u)$. It contracts to that $u$ is a minimizer.

Next, we claim that

$$
u_{+}=0 \quad \text { or } \quad u_{-}=0 .
$$

To prove the claim, we note that by (4.4), the equality holds in (4.5). As a consequence, the function $\tilde{u}$ defined previously is also a minimizer, so it is a solution to (1.4). Moreover, $\tilde{u}=|u|$. For contradiction, we assume that $u_{+} \neq 0$ and $u_{-} \neq 0$. Observe from the orthogonality of $u_{+}$and $u_{-}$that

$$
\left\|u_{+}\right\|_{H_{P+\mu}}^{2}-\left\|u_{+}\right\|_{p+1}^{p+1}+\left\|u_{-}\right\|_{H_{P+\mu}}^{2}-\left\|u_{-}\right\|_{p+1}^{p+1}=0 .
$$

Then either

$$
\left\|u_{+}\right\|_{H_{P+\mu}}^{2}-\left\|u_{+}\right\|_{p+1}^{p+1} \leq 0 \quad \text { or } \quad\left\|u_{-}\right\|_{H_{P+\mu}}^{2}-\left\|u_{-}\right\|_{p+1}^{p+1} \leq 0
$$


and we just may assume $\left\|u_{+}\right\|_{H_{P+\mu}}^{2}-\left\|u_{+}\right\|_{p+1}^{p+1} \leq 0$. Since $u_{+} \neq 0$, we can take $t \in(0,1]$ such that $\left\|t u_{+}\right\|_{H_{P+\mu}}^{2}-\left\|t u_{+}\right\|_{p+1}^{p+1}=0$. This implies

$$
\begin{aligned}
& \mathcal{I}\left(t u_{+}\right)=\left(\frac{1}{2}-\frac{1}{p+1}\right)\|t u\|_{H_{P+\mu}}^{2} \\
< & \left(\frac{1}{2}-\frac{1}{p+1}\right)\left\|u_{+}\right\|_{H_{P+\mu}}^{2}+\left(\frac{1}{2}-\frac{1}{p+1}\right)\left\|u_{-}\right\|_{H_{P+\mu}}^{2} \\
= & \mathcal{I}(u) .
\end{aligned}
$$

The strict inequality is due to the assumption that $u_{-} \neq 0$. Since $u$ is a minimizer, this makes a contradiction and the claim is proved. Finally, we have already seen in the proof of Theorem 1.1 that a nonnegative (nonpositive) minimizer is positive (negative) everywhere since the fundamental solution $\Phi_{P+\mu}$ of $P+\mu$ is strictly positive. This ends the proof.

\subsection{Uniqueness of a ground state}

Here, we prove Theorem 1.3. Throughout this subsection, we assume (H1)-(H4) on the symbol $P$ hold. We fix arbitrarily chosen $p \in(1, d /(d-s))$ and denote by $u_{0}$ the unique radial positive ground state to the original NLS,

$$
-\Delta u+u=|u|^{p-1} u .
$$

As mentioned in Section 3, the generalized NLS

$$
P(-\Delta) u+\epsilon u=|u|^{p-1} u
$$

is equivalently transformed to

$$
P_{\epsilon}(-\Delta) v+v=|v|^{p-1} v
$$

by the scaling $u(x)=\epsilon^{\frac{1}{p-1}} v(\epsilon x)$, where $P_{\epsilon}(\lambda):=\frac{1}{\epsilon} P(\epsilon \lambda)$. It is also straightforward to see that a ground state $u_{\epsilon} \in H_{P+\epsilon}$ to (4.8) corresponds to a ground state $v_{\epsilon} \in H_{P_{\epsilon}+1}$ to (4.9) by the scaling $u_{\epsilon}(x)=\epsilon^{\frac{1}{p-1}} v_{\epsilon}(\epsilon x)$ and vice versa. Thus, to prove Theorem 1.3, it is sufficient to show that a ground state $v_{\epsilon}$ to (4.9) is unique up to a translation for sufficiently small $\epsilon>0$.

Lemma 4.2 (Convergence). Let $v_{\epsilon} \in H_{P_{\epsilon}+1}$ be a positive ground state to (4.9). Then there exists $\left\{a_{\epsilon}\right\} \subset \mathbb{R}^{d}$ such that

$$
\lim _{\epsilon \rightarrow 0}\left\|v_{\epsilon}\left(\cdot-a_{\epsilon}\right)-u_{0}\right\|_{H_{P_{\epsilon}+1}}=0
$$


Proof. The lemma is a natural consequence of energy minimality of the ground states $v_{\epsilon}$ to (4.9). We refer to Proposition 2.3 in [8], with which their proof follows the exactly same lines. We omit the proof for avoiding the paper too lengthy.

Lemma 4.3 (Nondegeneracy). Let $\left\{v_{\epsilon}\right\} \subset H_{P_{\epsilon}+1}$ be a family positive ground state to (4.9) such that $\left\{v_{\epsilon}\right\}$ converges to $u_{0}$ in $H_{P_{\epsilon}+1}$ as $\epsilon \rightarrow 0$. Define the linearized operator by

$$
\mathcal{L}_{\epsilon}:=P_{\epsilon}(-\Delta)+1-p v_{\epsilon}^{p-1} \text {. }
$$

Then there exists a constant $\beta>0$ independent of small $\epsilon>0$ such that

$$
\beta\|g\|_{H_{P_{\epsilon}+1}} \leq\left\|\mathcal{L}_{\epsilon} g\right\|_{L^{2}}
$$

for any $g \in H_{P_{\epsilon}+1}$ which is $H_{P_{\epsilon}+1}$ orthogonal to $\partial_{x_{i}} v_{\epsilon}$ for each $i=1, \cdots, d$.

Proof. This lemma can be proved in the same spirit with Lemma 3.3 but one should take care on the change of function spaces from the radial function space $\left(W_{r}^{s, q} \rightarrow\right.$ $\left.\operatorname{span}\left\{\partial_{x_{i}} v_{\epsilon} \mid i=1, \cdots, d\right\}^{\perp}\right)$. This can be easily done by repeating the proof of Proposition 3.3 in [8]. We omit it.

Proof of Theorem 1.3. Let $\left\{v_{\epsilon}\right\},\left\{\tilde{v}_{\epsilon}\right\} \subset H_{P_{\epsilon}+1}$ be two families of positive radially symmetric ground states to (4.9). By Lemma 4.2, we may assume that both of $\left\{v_{\epsilon}\right\}$ and $\left\{\tilde{v}_{\epsilon}\right\}$ converge to $u_{0}$ in $H_{P_{\epsilon}+1}$ as $\epsilon \rightarrow 0$ by taking translations if necessary. This means that

$$
\lim _{\epsilon \rightarrow 0}\left\|v_{\epsilon}-\tilde{v}_{\epsilon}\right\|_{H_{P_{\epsilon}+1}}=0
$$

Let $\left\{a_{\epsilon}\right\} \subset \mathbb{R}^{d}$ be a family of vectors such that

$$
\left\|\tilde{v}_{\epsilon}\left(\cdot-a_{\epsilon}\right)-v_{\epsilon}\right\|_{H_{P_{\epsilon}+1}}^{2}=\min _{a \in \mathbb{R}^{d}}\left\|\tilde{v}_{\epsilon}(\cdot-a)-v_{\epsilon}\right\|_{H_{P_{\epsilon}+1}}^{2} .
$$

Then one has

$$
\left\langle\tilde{v}_{\epsilon}\left(\cdot-a_{\epsilon}\right)-v_{\epsilon}, \partial_{x_{i}} v_{\epsilon}\right\rangle_{H_{P_{\epsilon}+1}}=0 \text { for each } i=1, \cdots, d,
$$

since

$$
\begin{aligned}
0 & =\left.\nabla_{a}\right|_{a=a_{\epsilon}}\left\|\tilde{v}_{\epsilon}(\cdot-a)-v_{\epsilon}\right\|_{H_{P_{\epsilon}+1}}^{2}=\left.\nabla_{a}\right|_{a=a_{\epsilon}}\left\|\tilde{v}_{\epsilon}-v_{\epsilon}(\cdot-a)\right\|_{H_{P_{\epsilon}+1}}^{2} \\
& =-2\left\langle\tilde{v}_{\epsilon}-v_{\epsilon}\left(\cdot-a_{\epsilon}\right),\left(\nabla_{x} v_{\epsilon}\right)\left(\cdot-a_{\epsilon}\right)\right\rangle_{H_{P_{\epsilon}+1}}=-2\left\langle\tilde{v}_{\epsilon}\left(\cdot-a_{\epsilon}\right)-v_{\epsilon}, \nabla_{x} v_{\epsilon}\right\rangle_{H_{P_{\epsilon}+1}} .
\end{aligned}
$$

We redefine $\tilde{v}_{\epsilon}\left(\cdot-a_{\epsilon}\right)$ by $\tilde{v}_{\epsilon}$ so that $\tilde{v}_{\epsilon}$ is still a ground state, $\tilde{v}_{\epsilon}-v_{\epsilon}$ is orthogonal to $\partial_{x_{i}} v_{\epsilon}$ in $H_{P_{\epsilon}+1}$ and $\lim _{\epsilon \rightarrow 0}\left\|v_{\epsilon}-\tilde{v}_{\epsilon}\right\|_{H_{P_{\epsilon}+1}}=0$ by definition of $a_{\epsilon}$.

Let us define $r_{\epsilon}=\tilde{v}_{\epsilon}-v_{\epsilon}$. From Eq. (4.9), one has

$$
\left(P_{\epsilon}(-\Delta)+1\right) r_{\epsilon}=\left|v_{\epsilon}+r_{\epsilon}\right|^{p-1}\left(v_{\epsilon}+r_{\epsilon}\right)-\left|v_{\epsilon}\right|^{p-1} v_{\epsilon},
$$


so that by Lemma 4.3,

$$
\beta\left\|r_{\epsilon}\right\|_{H_{P_{\epsilon}+1}} \leq\left\|\mathcal{L}_{\epsilon} r_{\epsilon}\right\|_{L^{2}}=\left\|\left|v_{\epsilon}+r_{\epsilon}\right|^{p-1}\left(v_{\epsilon}+r_{\epsilon}\right)-\left|v_{\epsilon}\right|^{p-1} v_{\epsilon}-p v_{\epsilon}^{p-1} r_{\epsilon}\right\|_{L^{2}} .
$$

It is easy to see from (H3) that there exists a uniform constant $C>0$ independent of $\epsilon \in(0,1)$ such that

$$
\|u\|_{H^{s / 2}} \leq C\|u\|_{H_{P_{\epsilon}+1}} .
$$

Then we invoke Lemma 3.4 and Hölder inequality to obtain

$$
\begin{gathered}
\quad\left\|\left|v_{\epsilon}+r_{\epsilon}\right|^{p-1}\left(v_{\epsilon}+r_{\epsilon}\right)-\left|v_{\epsilon}\right|^{p-1} v_{\epsilon}-p v_{\epsilon}^{p-1} r_{\epsilon}\right\|_{L^{2}} \\
\leq \begin{cases}\left\|r_{\epsilon}\right\|_{H_{\epsilon}+1^{\prime}}^{p} & \text { if } 1<p \leq 2, \\
\left\|v_{\epsilon}+\left|r_{\epsilon}\right|\right\|_{H_{P_{\epsilon}+1}}^{p-2}\left\|r_{\epsilon}\right\|_{H_{P_{\epsilon}+1}}^{2}, & \text { if } 2<p .\end{cases}
\end{gathered}
$$

Combining this with (4.16) and using the fact that $\lim _{\epsilon \rightarrow 0}\left\|r_{\epsilon}\right\|_{H_{P_{\epsilon}+1}}=0$, we get $r_{\epsilon}=0$ for sufficiently small $\epsilon>0$. This shows $\tilde{v}_{\epsilon}=v_{\epsilon}$ and ends the proof.

\section{Acknowledgements}

This research of the first author was supported by Basic Science Research Program through the National Research Foundation of Korea (NRF) funded by the Ministry of Science and ICT (NRF-2020R1A2C4002615). This research of the second author was supported by Basic Science Research Program through the National Research Foundation of Korea (NRF) funded by the Ministry of Science and ICT (NRF-2020R1C1C1A01006415)

\section{References}

[1] H. Berestycki and P.-L. Lions, Nonlinear scalar field equations I. Existence of a ground state, Arch. Rational Mech. Anal., 82(4) (1983), 313-345.

[2] S. Bernstein, Sur les fonctions absolument monotones, Acta Math., 52(1) (1929), 1-66.

[3] J. Byeon, O. Kwon and J. Seok, Nonlinear scalar field equations involving the fractional Laplacian, Nonlinearity, 30(4) (2017), 1659-1681.

[4] S.-M. Chang, S. Gustafson, K. Nakanishi and T. P. Tsai, Spectra of linearized operators for NLS solitary waves, SIAM J. Math. Anal., 39(4) (2007/08), 1070-1111.

[5] X. Chang and Z.-Q. Wang, Ground state of scalar field equations involving a fractional Laplacian with general nonlinearity, Nonlinearity, 26(2) (2013), 479-494.

[6] W. Choi, Y. Hong and J. Seok, On critical and supercritical pseudo-relativistic nonlinear Schrödinger equations, Proc. Roy. Soc. Edinburgh Sect. A, 150(3) (2020), 1241-1263.

[7] W. Choi, Y. Hong and J. Seok, Optimal convergence rate and regularity of nonrelativistic limit for the nonlinear pseudo-relativistic equations, J. Funct. Anal., 274(3) (2018), 695-722.

[8] W. Choi, Y. Hong and J. Seok, Uniqueness and symmetry of ground states for higher-order equations, Cal. Var. Partial Differential Equations, 57(3) (2018). 
[9] V. Coti-Zelati and M. Nolasco, Existence of ground states for nonlinear, pseudo-relativistic Schrödinger equations, Rend. Lincei Mat. Appl., 22 (2011), 51-72.

[10] R. L. Frank and E. Lenzmann, Uniqueness of non-linear ground states for fractional Laplacians in R, Acta Math., 210(2) (2013), 261-318.

[11] R. L. Frank, E. Lenzmann and L. Silvestre, Uniqueness of radial solutions for the fractional Laplacian, Commun. Pure Appl. Math., 69(9) (2016), 1671-1726.

[12] F. Gazzola, H.-C. Grunau, and G. Sweers, Polyharmonic Boundary Value Problems, Positivity Preserving and Nonlinear Generalized Elliptic Equations in Bounded Domains, Lecture Notes in Mathematics, 1991. Springer-Verlag, Berlin, 2010. xviii+423 pp.

[13] B. Gidas, W. M. Ni and L. Nirenberg, Symmetry of positive solutions of nonlinear elliptic equations in $\mathbb{R}^{n}$, Mathematical Analysis and Applications, Part A, pp. 369-402, Adv. Math. Suppl. Stud., Academic Press, New York-London, 1981.

[14] M. K. Kwong, Uniqueness of positive solutions of $\Delta u-u+u^{p}=0$ in $\mathbb{R}^{n}$, Arch. Rational Mech. Anal., 105(3) (1989), 243-266.

[15] N. Laskin, Fractional Schrödinger equation, Phys. Rev. E, 66(5) (2002), 056108.

[16] E. Lenzmann, Uniqueness of ground states for pseudo-relativistic Hartree equations, Anal. PDE, 2 (2009), 1-27.

[17] E. H. Lieb and M. Loss, Analysis, Graduate Studies in Mathematics (American Mathematical Society, Providence, RI,1997), Vol. 14.

[18] E. H. Lieb and H. T. Yau, The Chandrasekhar theory of stellar collapse as the limit of quantum mechanics, Commun. Math. Phys., 112 (1987), 147-174.

[19] J.-J. Moreau, Décomposition orthogonale dún espace hilbertien selon deux cônes mutuellement polaires, C. R. Acad. Sci. Paris, 255 (1962), 238-240.

[20] S. Secchi, A generalized pseudorelativistic Schrödinger equation with supercritical growth, Commun. Contemp. Math., 21(8) (2019), 1850073.

[21] R. Schilling, R. Song, Z. Vondraček, Bernstein Functions, Theory and Applications, Second edition, De Gruyter Studies in Mathematics, 37. Walter de Gruyter \& Co., Berlin, 2012. xiv+410 pp.

[22] W. A. Strauss, Existence of solitary waves in higher dimensions, Commun. Math. Phys., 55(2) (1977), 149-162.

[23] M. Struwe, Variational Methods, Applications to Nonlinear Partial Differential Equations and Hamiltonian Systems, Springer-Verlag, Berlin, 1990. xiv+244 pp. 